\title{
Progranulin Mediates Caspase-Dependent Cleavage of TAR DNA Binding Protein-43
}

\author{
Yong-Jie Zhang, ${ }^{\star}$ Ya-fei Xu, ${ }^{1 \star}$ Chad A. Dickey, ${ }^{2}$ Emanuele Buratti, ${ }^{3}$ Francisco Baralle, ${ }^{3}$ Rachel Bailey, ${ }^{1}$ \\ Stuart Pickering-Brown, ${ }^{4}$ Dennis Dickson, ${ }^{1}$ and Leonard Petrucelli ${ }^{1}$ \\ ${ }^{1}$ Department of Neuroscience, Mayo Clinic College of Medicine, Jacksonville, Florida 32224, ${ }^{2}$ Department of Molecular Pharmacology and Physiology, \\ University of South Florida, Tampa, Florida 33612, ${ }^{3}$ International Centre for Genetic Engineering and Biotechnology, 34012 Trieste, Italy, and ${ }^{4}$ Division of \\ Regenerative Medicine, University of Manchester, Manchester M13 9PT, United Kingdom
}

TAR DNA binding protein-43 (TDP-43) is the pathologic substrate of neuronal and glial inclusions in frontotemporal lobar degeneration with ubiquitin-positive inclusions (FTDL-U) and in amyotrophic lateral sclerosis (ALS). Mutations in the progranulin gene (PGRN) have been shown to cause familial FTLD-U. The relationship between progranulin and TDP-43 and their respective roles in neurodegeneration is unknown. We report that progranulin mediates proteolytic cleavage of TDP-43 to generate $\sim 35$ and $\sim 25 \mathrm{kDa}$ species. Suppression of PGRN expression with small interfering RNA leads to caspase-dependent accumulation of TDP-43 fragments that can be inhibited with caspase inhibitor treatment. Cells treated with staurosporine also induced caspase-dependent cleavage and redistribution of TDP-43 from its nuclear localization to cytoplasm. Altered cleavage and redistribution of TDP-43 in cell culture models are similar to findings in FTLD-U and ALS. The results suggest that abnormal metabolism of TDP-43 mediated by progranulin may play a pivotal role in neurodegeneration.

Key words: caspase; apoptosis; frontotemporal lobar degeneration with ubiquitin-positive inclusions (FTDL-U); amyotrophic lateral sclerosis (ALS); TDP-43; progranulin

\section{Introduction}

Mutations in the gene for the secreted growth factor progranulin are responsible for a proportion of familial frontotemporal lobar degeneration with ubiquitin-positive inclusions (FTLD-U), the most common inherited form of frontotemporal dementia (Baker et al., 2006; Cruts et al., 2006). Mutations in the gene for progranulin (PGRN) almost all create null alleles, resulting in decreased progranulin protein or haploinsufficiency (Baker et al., 2006; Cruts et al., 2006). Subsequent to this finding, the major constituent protein in the inclusions of FTLD-U and amyotrophic lateral sclerosis (ALS) was identified (Neumann et al., 2007). The protein, a DNA binding protein involved in alternative exon splicing, TAR DNA binding protein-43 (TDP-43) (Ou et al., 1995; Buratti and Baralle, 2001; Buratti et al., 2001, 2005), undergoes a number of posttranslational modifications, including proteolytic cleavage (generating low-molecular-weight $\sim 25$ and 35 $\mathrm{kDa}$ species), phosphorylation, and ubiquitination. In both FTLD-U and ALS, TDP-43 translocates from its normal nuclear location into the cytoplasm, in which it forms disease-related aggregates, a process that possibly contributes to loss of normal

\footnotetext{
Received July 27, 2007; revised Aug. 15, 2007; accepted Aug. 15, 2007.

This work was supported by the Mayo Clinic Foundation and National Institute on Aging Grant R01-AG-02625101. We thank Dr. Mike Hutton, Matthew Baker, and Judith Dunmore for their help in the preparation of this manuscript.

*Y.-J.Z. and Y.-f.X. contributed equally to this work.

Correspondence should be addressed to Dr. Leonard Petrucelli, Mayo Clinic Jacksonville, 4500 San Pablo Road, Jacksonville, FL 32224. E-mail: petrucelli.leonard@mayo.edu.

D01:10.1523/JNEUROSCI.3421-07.2007

Copyright $\odot 2007$ Society for Neuroscience $\quad$ 0270-6474/07/2710530-05\$15.00/0
}

nuclear function (Neumann et al., 2007). Although evidence suggests that FTLD-U and ALS may have a common pathogenesis involving TDP-43 as a key mediator, interacting molecules or factors influencing TDP-43 cell biology have not been identified. Based on this information, we investigated whether progranulin is involved in TDP-43 processing (Neumann et al., 2007).

\section{Materials and Methods}

\section{Methods}

Cell culture and treatments. HeLa and $\mathrm{H} 4$ cells were grown in Opti-Mem plus $10 \%$ FBS and 1\% penicillin-streptomycin and passaged every $3-5 \mathrm{~d}$ based on $90 \%$ confluence. For PGRN small interfering RNA (siRNA) transfections, siRNA was predesigned by Qiagen (Valencia, CA) for Entrez accession number NM_001012479, and the sense sequence was $5^{\prime}$-r (GGCCACUCCUGCAUCUUUA) dTdT- $3^{\prime}$. siRNA experiments were performed in six-well plates. Final siRNA concentration (PGRN or a validated negative control siRNA) per well was 20 nм in Opti-Mem, with $4 \mu \mathrm{l}$ of siLentFect transfection reagent (Bio-Rad, Hercules, CA) used per well. This mixture was incubated in a final volume of $500 \mu \mathrm{l}$ for $20 \mathrm{~min}$ and then added to $40-50 \%$ confluent $\mathrm{HeLa}$ and $\mathrm{H} 4$ cells in six-well dishes plated the previous day for a final in-well volume of $2 \mathrm{ml}$. Seventy-two hours after transfection, cells were harvested for subsequent Western blot analysis in lysis buffer containing coimmunoprecipitation (Co-IP) buffer ( $50 \mathrm{~mm}$ Tris- $\mathrm{HCl}, \mathrm{pH} 7.4,1 \mathrm{~m} \mathrm{NaCl}, 1 \%$ Triton X-100, and $5 \mathrm{~mm}$ EDTA) plus $1 \%$ SDS, PMSF, and both a protease and phosphatase inhibitor mixture. For caspase inhibitor treatments, the cells were transfected with PGRN siRNA for $24 \mathrm{~h}$, and then the pan-caspase inhibitor Z-VADFMK ( $N$-benzyloxycarbonyl-Val-Ala-Asp-fluoromethyl ketone; EMD Chemicals, San Diego, CA) was added to cells for additional $48 \mathrm{~h}$ at a final concentration of $100 \mu \mathrm{M}$. Cell lysates were prepared as described above. For staurosporine treatment, $0.2 \mu \mathrm{M}$ staurosporine (Cell Signaling Tech- 
A.

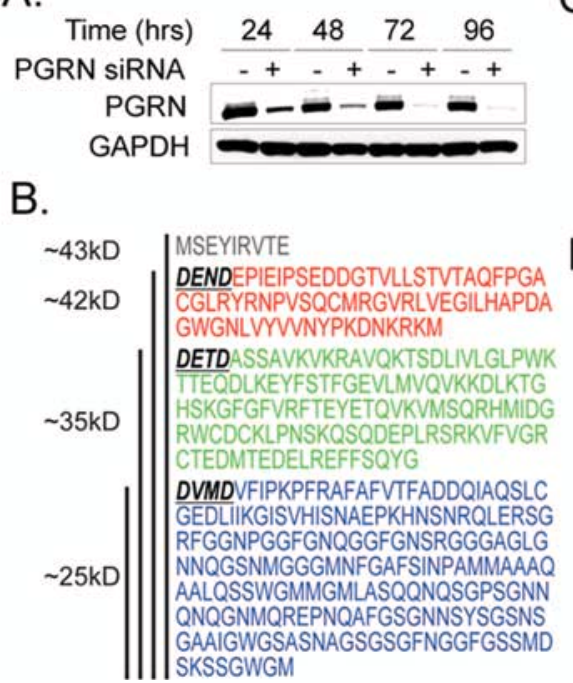

C.

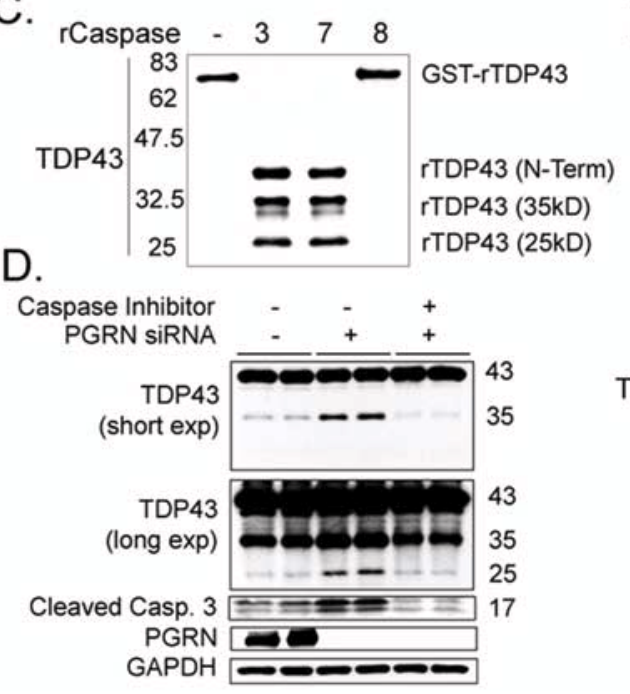

E.

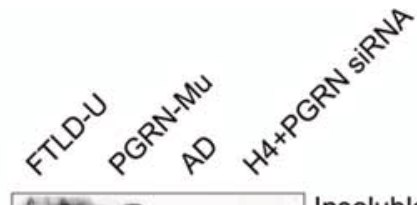

TDP43

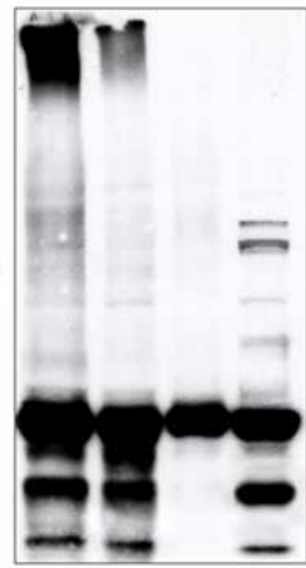

Insoluble

F.L. TDP43 $35 \mathrm{kD}$ Frag $25 \mathrm{kD}$ Frag

Figure 1. Proteolytic processing of TDP-43. A, Progranulin knockdown in H4 neuroglioma cell line using siRNA. B, Schematic representation of the amino acid sequence of TDP-43 (Entrez accession number NP_031401) depicting the three caspase-3 recognition motifs, DXXD (bold and underlined), cleavage of which would be predicted to generate fragments with approximate molecular weights of $42 \mathrm{kDa}$ (red), $35 \mathrm{kDa}$ (green), and $25 \mathrm{kDa}$ (blue). C, In vitro cleavage of recombinant TDP-43 protein. GST-tagged TDP-43 protein ( $2 \mu \mathrm{g}$ ) was incubated with purified cleaved caspase-3, caspase-7, or caspase-8 (2 U; Chemicon) for $4 \mathrm{~h}$. Samples were separated on 10\% SDS-PAGE and immunoblotted with rabbit anti-TDP-43 antibody. Similar results were obtained in duplicate experiments. $\boldsymbol{D}$, In vitro generation of pathologic TDP-43 by cells treated with PGRN siRNA but not by cells treated with control siRNA. Progranulin deficiency leads to an increase in cleaved caspase-3 activity. Treatment with a pan-caspase inhibitor suppresses progranulin-mediated TDP-43 cleavage and caspase-3 activity. Similar results were obtained in triplicate experiments. $\boldsymbol{E}$, Biochemical analyses of TDP-43 in sporadic and familial (PGRN mutant; Gly333ValfsX28) FTLD-U (Gass et al., 2006) and AD brains. Immunoblots of urea fractions from temporal cortex of FTLD-U patients and PGRN siRNA total cell extract with rabbit anti-TDP-43 antibody showed a pathological profile of TDP-43 similar to that in progranulin knockdown experiments but not in Alzheimer's disease or vascular dementia brains (data not shown). F.L., Full-length.

nology, Beverly, MA) was added to cells $3 \mathrm{~h}$ before the harvest. For time course experiments with siRNA treatment, the cells were treated with PGRN siRNA or control siRNA for $24,48,72$, or $96 \mathrm{~h}$, respectively. After treatment, cells were harvested for subsequent Western blot analysis.

Fractionation experiments. Briefly, cells or human tissue were lysed in a buffer containing Co-IP buffer plus PMSF and both a protease and phosphatase inhibitor mixture. After sonication, cells were centrifuged at $100,000 \times g$ at $4^{\circ} \mathrm{C}$ for $30 \mathrm{~min}$. Triton X-100-insoluble pellets were dissolved in the Co-IP buffer plus 1\% SDS, PMSF, and both a protease and phosphatase inhibitor mixture. The soluble and insoluble fractions were used in Western blot analysis. The urea fraction of human tissue was prepared as described previously (Neumann et al., 2007). Briefly, frozen gray matter from FTLD-U postmortem cortex of a patient with a known PGRN mutation was dissected and weighed. The tissue was extracted sequentially with low-salt buffer, high salt-Triton X-100 buffer, myelin floatation buffer, and Sarkosyl buffer. The Sarkosyl-insoluble materials were extracted in urea buffer and saved as urea. The urea fraction was used in Western blot analysis.

Western blot analysis. Protein concentrations of cells lysates or tissue homogenates were measured by a standard BCA assay (Pierce, Rockford, IL). The samples were then heated in Laemmli's buffer, and equal amounts of protein were loaded into 10 -well 10 or 4-20\% Tris-glycine gels (Novex, San Diego, CA). After transfer to nitrocellulose membranes, blots were blocked with 5\% nonfat dry milk in TBST (TBS plus $0.1 \%$ Triton X-100) for $1 \mathrm{~h}$ and then incubated with rabbit polyclonal TDP-43 antibody (1:1000; ProteinTech Group, Chicago, IL), rabbit polyclonal progranulin antibody (1:1000; Zymed Laboratories, South San Francisco, CA), rabbit polyclonal caspase- 3 antibody (1:1000; Cell Signaling Technology), or mouse monoclonal glyceraldehyde-3-phosphate dehydrogenase (GAPDH) antibody (1:5000; Biodesign International, Kennebunkport, ME) overnight at $4^{\circ} \mathrm{C}$. Membranes were washed three times for $10 \mathrm{~min}$ in TBST and then incubated with anti-mouse or anti-rabbit IgG conjugated to horseradish peroxidase (1:5000; Jackson ImmunoResearch, West Grove, PA) for $1 \mathrm{~h}$. Membranes were then washed three times for $10 \mathrm{~min}$, and protein expression was visualized by ECL treatment and exposure to film.
Immunofluorescence and confocal microscopy. HeLa or H4 cells grown on glass coverslips were treated with control or PGRN siRNA for $72 \mathrm{~h}$. $\mathrm{HeLa}$ or $\mathrm{H} 4$ cells were grown on glass coverslips for $48 \mathrm{~h}$, and then treated with 0.2 or $1 \mu \mathrm{M}$ staurosporine for $3 \mathrm{~h}$, respectively. After treatment with siRNA or staurosporine, the cells were fixed with ice-cold methanol at $-20^{\circ} \mathrm{C}$ for $5 \mathrm{~min}$ and permeabilized with PBS- $0.5 \%$ Triton X-100 for 10 min. After blocking with $5 \%$ BSA for $1 \mathrm{~h}$ at $37^{\circ} \mathrm{C}$, the cells were incubated overnight at $4^{\circ} \mathrm{C}$ with rabbit polyclonal TDP-43 antibody (1:2000), rabbit polyclonal progranulin antibody (1:250), mouse monoclonal $\beta$-actin antibody (1:2000; Sigma, St. Louis, MO), or rabbit polyclonal histone H3 antibody (1:100; Cell Signaling Technology), respectively. After washing, cells were incubated with the Oregon Green 488-conjugated goat antirabbit IgG secondary antibody (TDP-43; 1:1000 or progranulin and histone $\mathrm{H} 3 ; 1: 500)$ or Alexa 568-conjugated goat anti-mouse IgG secondary antibody ( $\beta$-actin; $1: 1000)$ at $37^{\circ} \mathrm{C}$ for $2 \mathrm{~h}$. Finally, Hoechst 33258 (1 $\mu \mathrm{g} / \mathrm{ml}$ ) was used to stain the nuclei. Images were obtained on a Zeiss (Thornwood, NY) LSM 510 META confocal microscope.

\section{In vitro caspase-3, caspase-7, and caspase- 8 assay}

Recombinant human glutathione S-transferase (GST)-TDP43 (2 $\mu \mathrm{g})$ was incubated separately with active human recombinant caspase-3, caspase-7, or caspase-8 (2 U; Chemicon, Temecula, CA) in reaction buffers containing $100 \mathrm{~mm} \mathrm{NaCl}, 50 \mathrm{~mm}$ HEPES, 10 mм DTT, 1 mм EDTA, $10 \%$ glycerol, and $0.1 \%$ CHAPS, $\mathrm{pH} 7.4$, at $37^{\circ} \mathrm{C}$ for $4 \mathrm{~h}$. Cleavage reactions were terminated by addition of $2 \times$ SDS loading buffer. Full-length or caspase-3-, caspase-7-, or caspase-8 treated recombinant GST-TDP43 was separated by $10 \%$ SDS-PAGE and subjected to Western blot analysis as above $(0.05 \mu \mathrm{g})$.

\section{Human brain tissue}

Sporadic and familial (PGRN mutant; Gly333ValfsX28) FTLD-U, Alzheimer's disease $(\mathrm{AD})$, and nondegenerative controls brain samples were obtained from Mayo Clinic Jacksonville Brain Bank as coded samples blinded to patient identifiers. The brain bank procedures are approved by institutional ethics committee. All cases had neuropathologic diagnostic studies according to previously published methods using currently ac- 


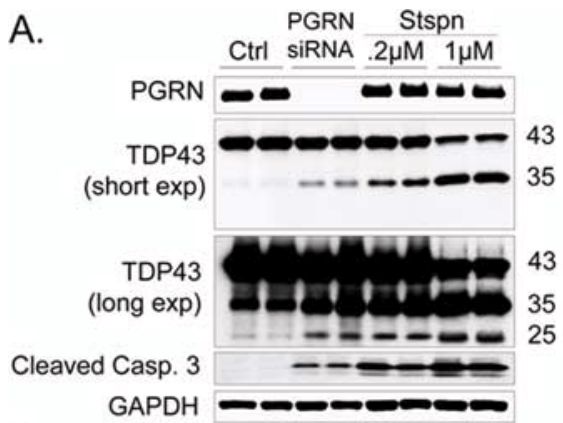

\section{B.}

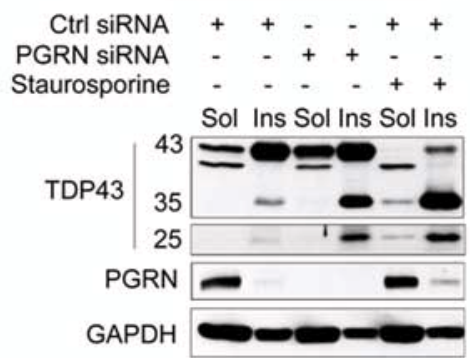

C.
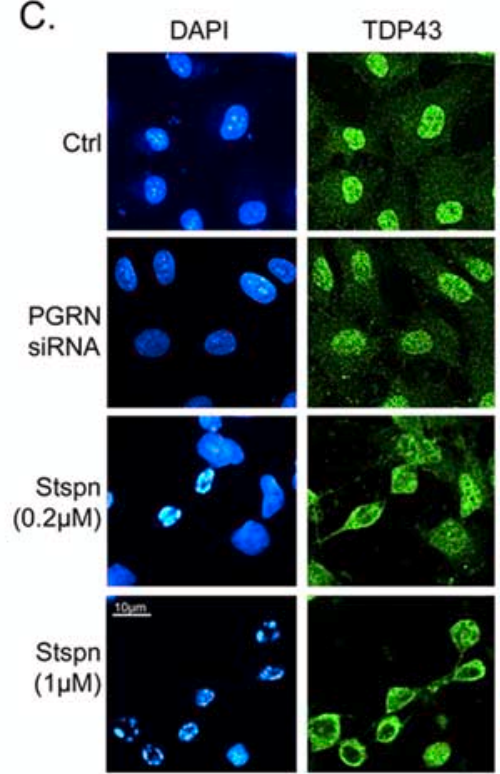
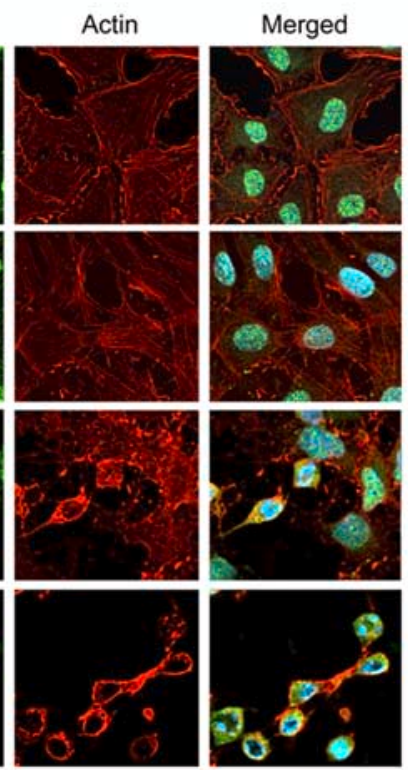

D. TDP43
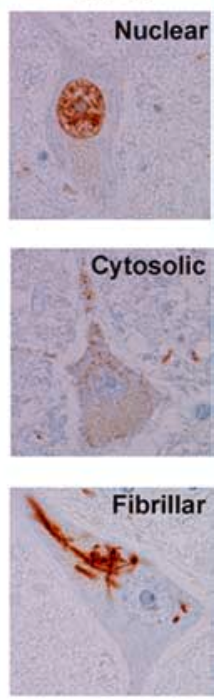

Figure 2. Cleavage, solubility, and cellular localization of TDP-43. A, H4 cells treated with staurosporine ( 0.2 and $1 \mu \mathrm{M}, 3 \mathrm{~h}$ ) and PGRN siRNA revealed reduction in full-length TDP-43, proteolytic cleavage of TDP-43, and increases in caspase-3 activity. Similar results were obtained in triplicate experiments. $\boldsymbol{B}$, Biochemical analyses of TDP-43 in HeLa cells. Immunoblots of cell extracts treated with PRGN siRNA or staurosporine with rabbit anti-TDP-43 antibody revealed the pathologic $\sim 25$ and $\sim 35 \mathrm{kDa}$ bands in Triton X-100-insoluble fractions. Similar results were obtained in triplicate experiments. C, Subcellular redistribution of TDP-43. Immunofluorescent staining for endogenous TDP-43 in H4 cells treated with control siRNA, PGRN siRNA, or staurosporine (0.2 and $1 \mu \mathrm{m}$ ) with rabbit anti-TDP-43 (green) or mouse monoclonal anti- $\beta$-actin (red) and examined by confocal microscopy. The nucleus is stained with $4^{\prime}, 6^{\prime}$-diamidino-2-phenylindole (DAPI; blue). Representative images of a field ( $n=5$ ) of one of three independent experiments from each culture. Scale bar, $10 \mu \mathrm{m}$. D, TDP-43 immunohistochemistry of motor neurons in ALS shows normal nuclear localization but no cytoplasmic labeling (top), early pathologic immunoreactivity characterized by diffuse granular cytoplasmic with no nuclear labeling (middle), and fully formed fibrillar cytoplasmic skein-like inclusions with no nuclear labeling (bottom). Pathologic redistribution of TDP-43 in cultured cells is similar to that in human disease. Magnification, $40 \times$. Ctrl, Control; Ins, insoluble; Sol, soluble; Stspn, staurosporine.

cepted diagnostic criteria (Dickson, 2005). Samples from temporal cortex were taken from frozen brain slabs and used for biochemical analyses; cases were matched for age, sex, duration of time in storage, and postmortem interval. For histologic studies, sections of medulla from a case with amyotrophic lateral sclerosis were processed for TDP-43 immunohistochemistry.

\section{Results}

\section{PGRN knockdown leads to caspase-dependent cleavage} of TDP-43

To model haploinsufficiency in cell culture models, two cell lines that have high endogenous levels of progranulin (HeLa epithelial cells and H4 neuroglioma cells) were treated with PGRN siRNA to selectively reduce progranulin protein levels. To determine the optimal time interval for in vitro knockdown, $\mathrm{H} 4$ neuroglioma cells (Fig. $1 A$ ) and HeLa cells (data not shown) were treated with either PGRN or control siRNA for 24, 48, 72, and $96 \mathrm{~h}$. As shown in Figure $1 A$, treatment with PGRN siRNA markedly reduced progranulin expression in a time-dependent manner with very little detectable progranulin protein observed on Western blots 72 and $96 \mathrm{~h}$ after treatment compared with control siRNA. Thus, for all subsequent studies, $72 \mathrm{~h}$ was used as the time point to evaluate effects of knockdown of progranulin. Endogenous progranulin was mostly present in the cytoplasm in these cell, consistent with in vivo data (supplemental Fig. 1, available at www. jneurosci.org as supplemental material) (Daniel et al., 2000; Mackenzie et al., 2006).

Analysis of the published amino acid sequence of TDP-43 revealed three potential caspase-3 cleavage consensus sites (DXXD) (Fig. $1 B$ ). Caspase-3 cleavage at these sites is predicted to produce $\sim 42,35$, and $25 \mathrm{kDa}$ fragments. Given that there were putative caspase- 3 cleavage sites in the TDP-43 amino acid se- quence, we wanted to determine whether caspase-3 and other caspases could cleave TDP-43 using an in vitro assay. Recombinant GST-tagged TDP-43 (N-terminal tag, full-length TDP-43) was incubated with or without purified cleaved caspase-3, caspase-7, and caspase- 8 for $4 \mathrm{~h}$. Western blots immunostained with an anti-rabbit TDP-43 antibody revealed cleavage of fulllength TDP- 43 by caspase- 3 and caspase- 7 but not caspase- 8 and generation of three distinct fragments of $\sim 42,35$, and $25 \mathrm{kDa}$ (Fig. 1C).

To explore a potential mechanism of progranulinknockdown-mediated cleavage of TDP-43, we examined the levels of total caspase- 3 under these conditions. As shown in Figure $1 \mathrm{D}$, cells treated with $P G R N$ siRNA had significantly increased levels of cleaved caspase-3 immunoreactivity compared with control siRNA, consistent with a previous report using neutralizing antibodies against progranulin (Liau et al., 2000).

After demonstration of specific in vitro knockdown of progranulin and its affect on caspase-3 activity, we investigated whether progranulin deficiency was associated with proteolytic processing of TDP-43. HeLa epithelial cells (Fig. 1D) and H4 neuroglioma cell lines (Fig. $2 A$ ) were treated with either control siRNA or PGRN siRNA for $72 \mathrm{~h}$. This experiment demonstrated that endogenous cellular TDP-43 was cleaved into similar $\sim 35$ and $\sim 25 \mathrm{kDa}$ fragments, similar to those found in our in vitro assay and in brains of FTLD-U (Fig. 1C,E). The generation of proteolytic fragments was inhibited by the caspase inhibitor Z-VAD (OMe)-FMK (Fig. 1D). Together, these data strongly suggest that suppression of $P G R N$ expression is sufficient to promote proteolytic cleavage of TDP-43 through a mechanism that implicates programmed cell death.

The $\sim 35$ and $\sim 25 \mathrm{kDa}$ fragments from these cell lysates had a 
A.

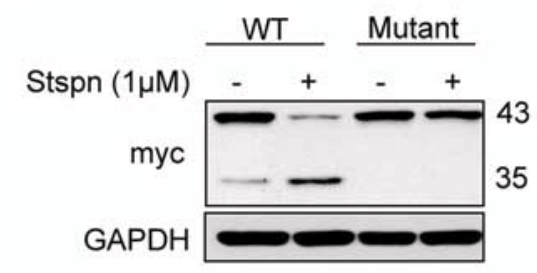

B.

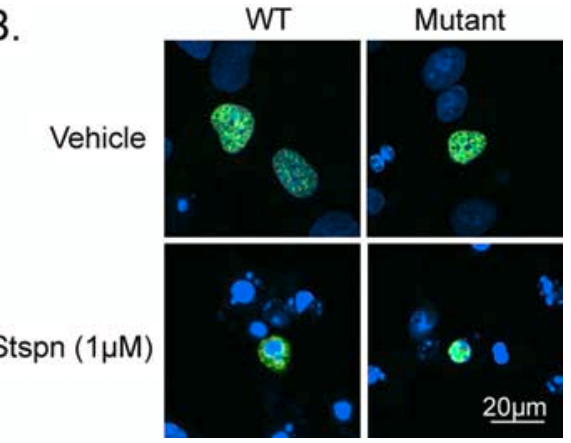

Figure 3. Cellular localization of caspase-resistant TDP-43. A, H4 cells transfected with either myc-tagged wild-type TDP-43 or myc-tagged double caspaseTDP-43 mutant (D89E, 35 kDa fragment; D219E, $25 \mathrm{kDa}$ fragment) in the presence or absence of staurosporine $(6 \mathrm{~h}, 1 \mu \mathrm{m})$. Immunoblots of cell extracts probed with mouse anti-myc antibody revealed resistance of the caspase mutant TDP-43 to proteolytic cleavage in cells treated with staurosporine. Similar results were obtained in duplicate experiments. $\boldsymbol{B}$, Subcellular redistribution of wild-type and caspase mutant TDP-43. Immunofluorescent staining for myc-tagged wild-type and caspase mutant TDP-43 in $\mathrm{H} 4$ cells treated with staurosporine ( $6 \mathrm{~h}, 1 \mu \mathrm{m})$ with mouse anti-TDP-43 (green) and examined by confocal microscopy. The nucleus is stained with $4^{\prime}, 6^{\prime}$-diamidino-2-phenylindole (DAPl; blue). Caspase mutant TDP-43 is retained in the nucleus in the presence of staurosporine. Scale bar, $20 \mu \mathrm{m}$. Representative images of one of three independent experiments from each culture. Stspn, Staurosporine; WT, wild type.

similar molecular mass and biochemical profile to TDP-43 extracted from brains of familial and sporadic FTLD-U with similar procedures (Fig. $1 E$ ). In contrast, TDP-43 from the brains of Alzheimer's disease (Fig. $1 E$ ) and vascular dementia (data not shown) did not contain these fragments.

\section{Redistribution of TDP-43 is dependent on caspase cleavage}

The evidence from these in vitro studies suggests that proteolytic cleavage of TDP-43 in FTLD-U may be mediated by caspases. To further confirm these results, $\mathrm{H} 4$ cells were exposed to staurosporine, a potent inducer of apoptosis and caspase- 3 activation. $\mathrm{H} 4$ cells treated with staurosporine at two doses $(0.2$ and $1 \mu \mathrm{M})$ exhibited a dose-dependent increase in cleavage of TDP-43 and caspase-3 activity (Fig. $2 \mathrm{~A}$ ) with a concomitant reduction in the major (full-length) TDP-43 band, most notable at the higher concentration. Treatment with PGRN siRNA showed similar results, although the amount of caspase- 3 activity was markedly less compared with that in the staurosporine treatment. Similar results were also found in HeLa cells (data not shown). To characterize the TDP-43 protein biochemically, HeLa cell lysates from control siRNA-, PGRN siRNA-, and staurosporine-treated conditions were separated into Triton X-100-soluble and -insoluble fractions and analyzed by Western blots. Whereas full-length TDP-43 protein was present in both soluble and insoluble fractions under control conditions, the $\sim 25$ and $\sim 35 \mathrm{kDa}$ bands were only detectable in Triton X-100-insoluble fractions of PGRN siRNA- and staurosporine-treated cells (Fig. 2 B). Similar results were also observed in human FTLD-U cases using the same buffer extraction (supplemental Fig. 2, available at www. jneurosci.org as supplemental material).

Next we explored the distribution of TDP-43 after treatment with PGRN siRNA or staurosporine $(0.2$ and $1 \mu \mathrm{M})$ as shown above. In contrast to untreated cells, which mostly exhibited nuclear localization of TDP-43 immunoreactivity (Fig. 2C), treatment of $\mathrm{H} 4$ cells with the higher dose of staurosporine exhibited a notable increase in cytoplasmic TDP-43 staining. In the absence of an antibody that is specific to neoepitopes generated by cleavage of TDP-43, it is not possible to state with certainty whether the cytoplasmic staining observed under these conditions is attributable to cleaved species or redistribution of full-length TDP-43 molecule. A marked redistribution was not observed

clusions (Fig. $2 D$, bottom).

with cells treated with PGRN siRNA or at the lower dose of staurosporine (although some redistribution was detected), perhaps reflecting the lower amount of caspase activity under these conditions. Another DNA-binding protein, histone $\mathrm{H} 3$, was localized to the nuclear compartment regardless of staurosporine treatment $(1 \mu \mathrm{M})$ (supplemental Fig. 3, available at www.jneurosci.org as supplemental material). This suggested that a general disruption of the nuclear membrane caused by staurosporine was unlikely to contribute to passive leakage of TDP-43 into the cytoplasm. Remarkably, this pattern of TDP-43 redistribution is also be observed in FTLD-U and ALS, in which vulnerable neurons (e.g., hypoglossal motor neurons in ALS) show progressive redistribution of TDP-43 from the nucleus (Fig. $2 D$, top) to cytoplasm (Fig. 2D, middle) and finally to fibrillar cytoplasmic in-

Next we explored whether the distribution of TDP-43 after treatment with staurosporine $(1 \mu \mathrm{M})$ as shown above was dependent on proteolytic cleavage of TDP-43. This was accomplished by using an artificial TDP-43 mutant (D89E, D219E) in which the consensus motif for caspase cleavage was mutated. As shown in Figure $3 A$, cells transfected with myc-tagged caspase mutant TDP-43 was resistant to proteolytic cleavage with staurosporine treatment. As expected, cells transfected with myc-tagged wildtype TDP-43 showed an increase in $35 \mathrm{kDa}$ species with a concomitant reduction in full-length TDP-43. In contrast to myctagged wild-type TDP-43 transfected cells, which mostly exhibited cytoplasmic localization of TDP-43 immunoreactivity with staurosporine treatment (Fig. $3 B$ ), treatment of myc-tagged mutant caspase TDP-43 cells at the same dose of staurosporine exhibited a notable nuclear TDP-43 staining. This result strongly suggests that the redistribution of TDP-43 (full-length and cleaved species) is initiated by caspase-induced TDP-43 cleavage and necessary for redistribution.

\section{Discussion}

The identification of TDP-43 as the major component of the neuronal and glial inclusions in FTLD-U and ALS, and the determination that haploinsufficiency of progranulin leads to FTLD-U, are pivotal findings for advancing our understanding of the dysfunctional pathways underlying these disorders (Neumann et al., 2007). The results herein demonstrate for the first time that decreases in progranulin can lead to pathological processing of TDP-43 by caspase-3, providing clear, and most importantly, testable hypotheses to facilitate greater understanding of the pathological processes contributing to FTLD-U and related disorders.

A number of similarities between our cell culture system and human cases of FTLD-U are notable. Activation of caspase- 3 is consistent with previous reports of activated caspase- 3 immunoreactivity in both FTLD-U and ALS (Martin, 1999; Su et al., 2000). The fragmentation of TDP-43 into 25 and $35 \mathrm{kDa}$ proteolytic species and changes in its solubility are also similar to biochemical properties of TDP-43 in brains of FTLD-U and ALS. The redistribution of TDP-43 from the nucleus to cytoplasm is 
yet another similarity between the cell culture model and human disease.

Although pharmacological suppression of caspase-3 is likely not an appropriate therapeutic strategy for FTLD-U and ALS (Thornberry and Lazebnik, 1998), our cell culture models provide screening tools for the identification of compounds that specifically prevent pathological fragmentation and redistribution of TDP-43 without affecting programmed cell death.

One key question that needs to be addressed is why reduction of progranulin leads to an increase in caspase- 3 activation and TDP-43 cleavage. It has been demonstrated that progranulin binds to secretory leukocyte protease inhibitor (SLPI), and this inhibits the elastase-mediated proteolysis of progranulin (Zhu et al., 2002). Therefore, it is conceivable that SLPI also inhibits caspase-3 as part of a progranulin/SLPI/TDP-43/caspase- 3 complex. Presumably, reduced levels of progranulin will lead to a reduction of SLPI within this putative complex, and this in turn frees caspase- 3 activity to cleave TDP-43 into the observed fragments. Furthermore, it remains to be established whether the 25 $\mathrm{kDa}$ fragment of TDP-43 is more fibrillogenic (a common feature of main proteins found within neuronal inclusions in neurodegenerative disease) than the full-length protein, and this contributes to its accumulation.

In summary, we demonstrated that reduction of progranulin is associated with proteolysis, change in solubility, and subcellular redistribution of TDP-43 similar to changes in FTLD-U and ALS. In addition, we have for the first time identified caspases (caspase-3 and caspase-7) as at least one class of proteases responsible for this cleavage. These data provide much needed insight into mechanisms linking progranulin and TDP-43 in disorders associated with TDP-43-immunoreactive inclusion bodies.

\section{References}

Baker M, Mackenzie IR, Pickering-Brown SM, Gass J, Rademakers R, Lindholm C, Snowden J, Adamson J, Sadovnick AD, Rollinson S, Cannon A, Dwosh E, Neary D, Melquist S, Richardson A, Dickson D, Berger Z, Eriksen J, Robinson T, Zehr C, et al. (2006) Mutations in progranulin cause tau-negative frontotemporal dementia linked to chromosome 17. Nature 442:916-919.

Buratti E, Baralle FE (2001) Characterization and functional implications of the RNA binding properties of nuclear factor TDP-43, a novel splicing regulator of CFTR exon 9. J Biol Chem 276:36337-36343.

Buratti E, Dork T, Zuccato E, Pagani F, Romano M, Baralle FE (2001) Nuclear factor TDP-43 and SR proteins promote in vitro and in vivo CFTR exon 9 skipping. EMBO J 20:1774-1784.

Buratti E, Brindisi A, Giombi M, Tisminetzky S, Ayala YM, Baralle FE (2005) TDP-43 binds heterogeneous nuclear ribonucleoprotein A/B through its
C-terminal tail: an important region for the inhibition of cystic fibrosis transmembrane conductance regulator exon 9 splicing. J Biol Chem 280:37572-37584.

Cruts M, Gijselinck I, van der Zee J, Engelborghs S, Wils H, Pirici D, Rademakers R, Vandenberghe R, Dermaut B, Martin JJ, van Duijn C, Peeters K, Sciot R, Santens P, De Pooter T, Mattheijssens M, Van den Broeck M, Cuijt I, Vennekens K, De Deyn PP, et al. (2006) Null mutations in progranulin cause ubiquitin-positive frontotemporal dementia linked to chromosome 17q21. Nature 442:920-924.

Daniel R, He Z, Carmichael KP, Halper J, Bateman A (2000) Cellular localization of gene expression for progranulin. J Histochem Cytochem 48:999-1009.

Dickson DW (2005) Required techniques and useful molecular markers in the neuropathologic diagnosis of neurodegenerative diseases. Acta Neuropathol (Berl) 109:14-24.

Gass J, Cannon A, Mackenzie IR, Boeve B, Baker M, Adamson J, Crook R, Melquist S, Kuntz K, Petersen R, Josephs K, Pickering-Brown SM, GraffRadford N, Uitti R, Dickson D, Wszolek Z, Gonzalez J, Beach TG, Bigio E, Johnson N, et al. (2006) Mutations in progranulin are a major cause of ubiquitin-positive frontotemporal lobar degeneration. Hum Mol Genet 15:2988-3001.

Liau LM, Lallone RL, Seitz RS, Buznikov A, Gregg JP, Kornblum HI, Nelson SF, Bronstein JM (2000) Identification of a human glioma-associated growth factor gene, granulin, using differential immuno-absorption. Cancer Res 60:1353-1360.

Mackenzie IR, Baker M, Pickering-Brown S, Hsiung GY, Lindholm C, Dwosh E, Gass J, Cannon A, Rademakers R, Hutton M, Feldman HH (2006) The neuropathology of frontotemporal lobar degeneration caused by mutations in the progranulin gene. Brain 129:3081-3090.

Martin LJ (1999) Neuronal death in amyotrophic lateral sclerosis is apoptosis: possible contribution of a programmed cell death mechanism. J Neuropathol Exp Neurol 58:459-471.

Neumann M, Kwong LK, Truax AC, Vanmassenhove B, Kretzschmar HA, Van Deerlin VM, Clark CM, Grossman M, Miller BL, Trojanowski JQ, Lee VM (2007) TDP-43-positive white matter pathology in frontotemporal lobar degeneration with ubiquitin-positive inclusions. J Neuropathol Exp Neurol 66:177-183.

Ou SH, Wu F, Harrich D, Garcia-Martinez LF, Gaynor RB (1995) Cloning and characterization of a novel cellular protein, TDP-43, that binds to human immunodeficiency virus type 1 TAR DNA sequence motifs. J Virol 69:3584-3596.

Su JH, Nichol KE, Sitch T, Sheu P, Chubb C, Miller BL, Tomaselli KJ, Kim RC, Cotman CW (2000) DNA damage and activated caspase-3 expression in neurons and astrocytes: evidence for apoptosis in frontotemporal dementia. Exp Neurol 163:9-19.

Thornberry NA, Lazebnik Y (1998) Caspases: enemies within. Science 281:1312-1316.

Zhu J, Nathan C, Jin W, Sim D, Ashcroft GS, Wahl SM, Lacomis L, Erdjument-Bromage H, Tempst P, Wright CD, Ding A (2002) Conversion of proepithelin to epithelins: roles of SLPI and elastase in host defense and wound repair. Cell 111:867-878. 